\title{
Tensile, creep, and fatigue behaviors of 3D printed acrylonitrile butadiene styrene
}

\author{
Hanyin Zhang ${ }^{1}$, Linlin Cai ${ }^{1}$, Michael Golub ${ }^{1}$, Yi Zhang ${ }^{1}$, Xuehui Yang ${ }^{1}$, Kate Schlarman², Jing \\ Zhang $^{1^{*}}$ \\ ${ }^{1}$ Department of Mechanical Engineering, Indiana University- Purdue University Indianapolis, \\ Indianapolis, IN 46202 \\ ${ }^{2}$ Decatur County Community Schools, Greensburg, IN 47240 \\ Corresponding author: jz29@iupui.edu
}

\begin{abstract}
Acrylonitrile butadiene styrene (ABS) is a widely used thermoplastics in 3D printing. However, there is a lack of thorough investigation of the mechanical properties of $3 \mathrm{D}$ printed ABS components, including orientation-dependent tensile strength, and creep fatigue properties. In this work, a systematic characterization is conducted on the mechanical properties of 3D printed ABS components. Specifically, the effect of printing orientation on the tensile and creep properties is investigated. The results show that, in tensile tests, the $0^{\circ}$ printing orientation has the highest Young's modulus of $1.81 \mathrm{GPa}$, and ultimate strength of $224 \mathrm{MPa}$. In the creep test, the $90^{\circ}$ printing orientation has the lowest $k$ value of 0.2 in the plastics creep model, suggesting $90^{\circ}$ is the most creep resistant direction. In the fatigue test, the average cycle number under load of $30 \mathrm{~N}$ is 3796 cycles. The average cycle number decreases to 128 cycles when the load is $60 \mathrm{~N}$. Using the Paris law, with an estimated crack size of $0.75 \mathrm{~mm}$, and stress intensity factor is varied from 352 to 700 $N \sqrt{m}$, the derived fatigue crack growth rate is $0.0341 \mathrm{~mm} /$ cycle. This study provides important mechanical property data that is useful for applying 3D printed ABS in engineering applications.
\end{abstract}

Keywords: Acrylonitrile Butadiene Styrene; ABS; additive manufacturing; 3D printing; printing orientation; tensile; creep; fatigue.

This is the author's manuscript of the article published in final edited form as:

Zhang, H., Cai, L., Golub, M., Zhang, Y., Yang, X., Schlarman, K., \& Zhang, J. (2018). Tensile, Creep, and Fatigue Behaviors of 3D-Printed Acrylonitrile Butadiene Styrene. Journal of Materials Engineering and Performance, 27(1), 57-62. 


\section{Introduction}

Acrylonitrile Butadiene Styrene (ABS) is a commonly used thermoplastic in 3D printing, where acrylonitrile can provide chemical resistance and impact resistance, butadiene provides toughness and impact resistance, and styrene gives rigidity and easy post-processing [1]. Therefore, ABS has become a common material in 3D printing due to its various advantages, including heat resistance, low-temperature-impact resistance, glossy surface, easy coloring [2].

Previous research has been done to study the effects of altering process parameters on the physical properties of the printed object, and process parameter effect on the dimensional accuracy and surface finish [3], and improving the compressive strength [4]. In this study, the research is focused on the effect of printing orientation on the tensile strength of 3D printed specimens. We have previously studied the effect of printing orientation on tensile strength of 3D printed ABS [5]. Brindley et al. measured the tensile properties of polycarbonate and ABS specimens in edge, upright printed orientations [6]. Hossain et al. examined how printing parameters, including orientation, raster angle and width, contour width, to improve the tensile properties [7]. Moore et al. studied how cross-section area and surface finish affect the fatigue life [8].

Although previous efforts, there is a lack of a systematic characterization of the mechanical properties of 3D printed ABS components. This work is aimed at conducting a thorough investigation of the mechanical properties of 3D printed ABS components, including orientationdependent tensile strength, and creep fatigue properties. The paper is arranged as follows. The experimental details are presented in Section 2, including 3D printing specimens and mechanical testing. The results and discussion are given in Section 3. Section 4 summarizes the results.

\section{Experimental details}

\subsection{D printing of ABS specimens}

\subsubsection{Tensile testing specimens}

The geometry of tensile specimens is designed in accordance with the specifications outlined in ASTM D-638 for the Type IV tensile specimens [9], as shown in Fig. 1. The 3D printer used is a Dimension SST 3D printer in conjunction with CatalystEx software (Stratasys Ldt., Eden Prairie, $\mathrm{MN})$. The printing parameters are as follows: melting temperature in a range of $220-230^{\circ} \mathrm{C}$, nozzle 
diameter $0.5 \mathrm{~mm}$, printing speed of $30 \mathrm{~mm} / \mathrm{s}$, layer height $0.1 \mathrm{~mm}$, internal contour with $100 \%$ dense packing accumulation mode.

To understand the effect of printing orientation on the mechanical properties of the 3D printed ABS specimens, three printing orientations, $0^{\circ}, 45^{\circ}$, and $90^{\circ}$, in x-y plane are selected. Printing orientation refers to where in the virtual $\mathrm{x}, \mathrm{y}, \mathrm{z}$ coordinate system of the 3D printer's software that the model is placed [10]. $0^{\circ}$ refers the specimen printed along the $x$-axis. $45^{\circ}$ refers the specimen placed between the $\mathrm{x}$-axis and y-axis. And $90^{\circ}$ refers the specimen printed along the y-axis. Printing orientation defines how a specimen is printed on the platform, which eventually can affect the strength and other properties of the specimen.

\subsubsection{Creep testing specimens}

The creep specimens used in this study are designed based on the recommendation of the manufacturer of the creep testing tester (SM106 Creep Measurement Apparatus, TecQuipment Ltd, Nottingham, United Kingdom), as shown in Fig. 2.

Similar to the tensile testing, to understand the effect of printing orientation on the mechanical properties of the $3 \mathrm{D}$ printed $\mathrm{ABS}$ specimens, three printing orientations, $0^{\circ}, 45^{\circ}$, and $90^{\circ}$, in $\mathrm{x}-\mathrm{y}$ plane are selected, as shown in Fig. 2b.

\subsubsection{Fatigue testing specimens}

The fatigue testing specimens are designed in accordance with the fatigue testing machine manufacturer (MT 3012-E, TERCO Material Testing Equipment, Sweden). The specimens are printed using a He3D single extruder Delta 180 3D printer.

\subsection{Mechanical testing of 3D printed ABS specimens}

\subsubsection{Tensile testing}

The tensile testing follows the ASTM D-638 [9] using a MTS universal testing machine MTS QTest/5 Universal Testing Machine (MTS, Eden Prairie, MN), with an extensometer 634.12E-54. The tensile rate applied is $0.2 \mathrm{in} / \mathrm{min}(0.0847 \mathrm{~mm} / \mathrm{s})$. The ABS specimens are pulled till fracture, 
and the load and elongation are recorded. Three samples of each orientation were tested to derive the average and deviation of the property values.

\subsubsection{Creep testing}

The creep measurement apparatus (SM106 Creep Measurement Apparatus, TecQuipment Ltd, Nottingham, United Kingdom) is used, as shown in Fig. 4. In order to obtain the creep strain versus time creep curve, the 3D printed specimens are applied with a small and constant load, 560 grams of weight. Fig. 4 shows one of the specimens loaded on the apparatus.

\subsubsection{Fatigue testing}

The fatigue test is conducted using a rotatory fatigue tester (MT 3012-E, TERCO Material Testing Equipment, Sweden). It applies reversing stress by employing a cantilever load on the specimens through rotating about its longitudinal axis. Hence the stress at any point on the surface of the cantilever varies sinusoidal. The motor drives the specimen to rotate at a speed of 3000 RPM (revolutions per minute), power supply provided is $200 \mathrm{~V}$ single phase.

The loading is applied through placing a hanging weight on the test piece. A spring balance measures the loading value. The hanging weight does not move, but the sample is rotated. The specimen is subjected to symmetric cyclic bending stress. When the sample fatigue fractures, the machine stops due to sudden load change, and the cycle number is recorded.

\section{Results and discussion}

\subsection{Tensile testing results}

The stress-strain curves of tensile bars in different printing orientations are plotted in Fig. 5. The figure shows both brittle and ductile material characteristics, which $0^{\circ}$ printing orientation has higher ultimate stress and yielding stress compared with the $45^{\circ}$ and $90^{\circ}$ printing orientations. As shown in Table 1, the Young's modulus for the $0^{\circ}, 45^{\circ}$, and $90^{\circ}$ orientations are $1.81 \mathrm{GPa}, 1.80 \mathrm{GPa}$, 
and $1.78 \mathrm{GPa}$, respectively. A previous study also shows the $0^{\circ}$ has the advantage on tensile properties among specimens printed in flat, edge and upright directions [6].

Table 1: Averaged mechanical strength as a function of printing orientation

\begin{tabular}{|l|l|l|l|}
\hline Printing orientation & $0^{\circ}$ & $45^{\circ}$ & $90^{\circ}$ \\
\hline Young's modulus(GPa) & $1.81+/-0.10$ & $1.80+/-0.11$ & $1.78+/-0.13$ \\
\hline Ultimate strength (MPa) & $22.4+/-0.1$ & $20.7+/-0.1$ & $19.0+/-0.2$ \\
\hline
\end{tabular}

The main reason that the $0^{\circ}$ has a relativelyhigher ultimate strength is that the loading direction during the tensile test is aligned with how the sample being printed during the process. A previous study also shows that average the Young's modulus of 3D printed ASTM D638 bar is $1.8 \mathrm{GPa}$, which is very close to our result [11]. This result indicates the best printing orientation is $0^{\circ}$ when trying to achieve higher ultimate strength in applications. A previous work has shown that the tensile strength of 3D printed specimen has similar strength, but inner microstructure has caused different time before fracture [12].

Stratasys allows users to define how the object being printed on the platform. As shown in Fig. 6 , there is a defined pattern how each layer is angled differently among $0^{\circ}, 45^{\circ}$, and $90^{\circ}$ printing orientations. Fig. 6 is a frequency chart showing the layer angles repeat about every five layers. From Fig. 5, we can see that each layer from stress axis is forming a sine wave.

For the sample morphology, as an example, Fig. 7 shows the $0^{\circ}$ printed tensile specimen before and after the tensile test, along with the detailed cross sectional view of the fracture surface. It is clear to see all the layers from layer 1 to layer 10 . When each layer is built, the printer automatically prints an enclosure of the border.

The detailed cross sectional view of the fracture surface is given in Fig. 7c. The smeared surface suggests a partial ductile fracture. The 10 slices or printing layers are visible from the left side of the fracture surface. Among these 10 layers, their orientations vary, resulting in different facture patterns. The orientation of each slice is increased with a finite angle, so the filaments partially overlap each other in order to achieve maximum contact surface strengths. 


\subsection{Creep testing results}

As shown in Fig. 4, during the creep test, a few horizontal cracks developed in the specimen as the result of stress. The creep curves for the three different angles of printing orientations material are determined. As shown in Fig. 8, three creep phases, primary, secondary, and ternary, are evident in all three curves, with a dominating secondary creep phase.

Polymers consist of chain-like molecules that are tangled. The creep occurs by chains untangling and slipping. However the 3D printed specimen created by string of melted ABS material, therefore, its composite-like structure does not provide as many tangled chain as normal polymers. The following equation is the most commonly used in analyze plastic creep model of empirical equation [13]:

$$
\varepsilon=\varepsilon_{0}+B \sigma^{m} t^{k}
$$

where $\varepsilon$ is the tensile creep strain after a time $t, \sigma$ is the applied creep stress, $\varepsilon_{0}$ is the instantaneous loading, and B, $\mathrm{m}, \mathrm{k}$ are constants for a given materials. A plot of $\log \varepsilon$ against $\log \mathrm{t}$ will therefore be linear and the slope will give the value of exponent $k$. In this work, we have calculated three different $k$ values because of the variation of printing orientation, as given in Table 2 .

Table 2: Calculated $k$ values verses printing orientation

\begin{tabular}{|l|l|l|l|}
\hline Printing orientation & 00 & 450 & 900 \\
\hline$k$ (average) & 0.455 & 0.243 & 0.200 \\
\hline
\end{tabular}

A large $k$ value means the material has a low creep resistance. Therefore, the $90^{\circ}$ specimen has the highest creep resistance among all orientations, and the $0^{\circ}$ has the lowest creep resistance. The results are also consistent with Fig. 8.

In creep test, $90^{\circ}$ is the most creep resistant orientation, while in tensile test, $0^{\circ}$ has the highest tensile properties. This is due to the fact that the creep test specimens are thin with only two or three layers. The tensile testing specimens are much thicker with ten layers. The tensile test specimens were prepared following the ASTM standard. The creep test specimens were prepared 
based on the recommendation of the creep tester manufacturer. Therefore, they have different dimensions, including their thickness. In summary, the creep specimens are more sensitive to the printing orientation as shown in Fig. 8, but the tensile specimens are not much sensitive, as shown in Figs. 5 and 6.

\subsection{Fatigue testing results}

The cycle number of material experiences under alternating load before failure is called fatigue life $N$. The maximum number of different cyclic stress tests obtained by fatigue test as well as the maximum stress curve can be plotted as $\sigma \max -N$ curve, commonly referred to as $S-N$ curve. Fig. 9 shows the $S-N$ curve of the 3D printed ABS specimens, with the calculated mean and standard deviation values.

The cross-sectional views of the broken fatigue specimens are also analyzed in Fig. 10. All specimens break at the smallest cross sectional region as shown in Fig. 3 because it is the location where the maximum stress is applied. From Fig. 10, it can clearly see both static fracture and fatigue fracture cause the fracture in the fatigue testing specimens. Static fracture is based on one load in the relatively white color part of the specimen center. Outside of the center region, it is fatigue fracture, which appears to be uneven surface compared to static fracture. It is also evident that as the load increases from $30 \mathrm{~N}$ (Fig. 10a) from $60 \mathrm{~N}$ (Fig. 10d), in general, the white region fraction increases, suggesting increasing contribution from static fracture.

To quantify the fatigue behavior, the following fatigue equation [19] is applied:

$$
\sigma_{a}=A N_{f}^{B}
$$

where both $A$ and $B$ are fitting constants.

In order to derive the fitting parameters in Eq. (2), the S-N curve is plotted in a log-log scale to obtain $\log \sigma$ vs. $\log N f$, as shown in Fig. 9. Using the slope and intersection, the derived A is 1336 $\mathrm{MPa}$, and $\mathrm{B}$ is -0.205 . It is noted that the specimens are not showing any thermal melting effect 
because of the short amount of test running time. Therefore, the calculation is based on no heating assumption.

The microstructure of the printed specimens also affects the fatigue behavior. The fatigue limit is not as constant for the material, but is dependent on other factors such as the type of load, volume of material, and surface finish etc. [8]. The crack propagation is analyzed by applying the Paris law, where the fatigue crack growth can be calculated using the following equation [19]:

$$
\frac{d a}{d N}=C(\Delta K)^{n}
$$

where $K$ is stress intensity factor, $C$ is material constant, $N$ is cycle number, and da/dN is $\mathrm{m} /$ cycle.

With an estimated crack size of $0.75 \mathrm{~mm}$ in Fig. 10, and the Paris law equation, the stress intensity factor is varied from 352 to $700 M N \sqrt{m} M N$. Therefore, the derived fatigue crack growth rate is $0.0341 \mathrm{~mm} /$ cycle.

\section{Conclusions}

Based on the tensile, creep, and fatigue testing of $3 \mathrm{D}$ printed ABS specimens at $0^{\circ}, 45^{\circ}$, and $90^{\circ}$, the following conclusions can be made:

1. In tensile test, the $0^{\circ}$ orientation has the highest mechanical properties, as it has the largest Young's modulus and ultimate strength, on average at $1.81 \mathrm{GPa}$ and $224 \mathrm{MPa}$ respectively.

2. In the creep test, the 90 o printing orientation has the lowest $k$ values, which is $\sim 0.2$, suggesting it has the best creep resistance properties.

3. In the fatigue test, the average cycle number under the load of $30 \mathrm{~N}$ is 3796 cycles. The average cycle number is decreased to 128 cycles when the load is $60 \mathrm{~N}$.

4. In the fatigue test, with an estimated crack size of $0.75 \mathrm{~mm}$, the derived fatigue crack growth rate is $0.0341 \mathrm{~mm} /$ cycle. 


\section{Acknowledgements}

J. Z. acknowledges the support from the Walmart Foundation (Project title: Optimal Plastic Injection Molding Tooling Design and Production through Advanced Additive Manufacturing). Linlin Cai is supported by the CTEE (Commitment to Engineering Excellence Research Fund) research program and MURI (Multidisciplinary Undergraduate Research Institute) program at Indiana University- Purdue University Indianapolis. Kate Schlarman is supported by NSF (award number: EEC 1406995).

\section{References}

1. Acrylonitrile butadiene styrene. Available from: https://en.wikipedia.org/wiki/Acrylonitrile_butadiene_styrene (accessed August 31, 2017).

2. 3D Printer Materials Guide. Available from: https://3dprinting.com/materials/ (accessed August 31, 2017).

3. Kumar, P., I. Ahuja, and R. Singh, Application of fusion deposition modelling for rapid investment casting-a review. International Journal of Materials Engineering Innovation, 2012. 3(3-4): p. 204-227.

4. Sood, A.K., R.K. Ohdar, and S.S. Mahapatra, Experimental investigation and empirical modelling of FDM process for compressive strength improvement. Journal of Advanced Research, 2012. 3(1): p. 81-90.

5. Cai, L., et al., Effect of Printing Orientation on Strength of 3D Printed ABS Plastics, in TMS 2016: 145th Annual Meeting \& Exhibition: Supplemental Proceedings. 2016, John Wiley \& Sons, Inc. p. 199-204.

6. Brindley, P., et al., Preliminary investigation into the mechanical properties of stratasys polycarbonate and m30 abs materials. 2008, Loughborough University.

7. Hossain, M.S., et al. Improving tensile mechanical properties of FDM-manufactured specimens via modifying build parameters. in International Solid Freeform Fabrication Symposium: An Additive Manufacturing Conference. Austin, TX. 2013.

8. Moore, J.P. and C.B. Williams, Fatigue properties of parts printed by PolyJet material jetting. Rapid Prototyping Journal, 2015. 21(6): p. 675-685.

9. ASTM D638-14, Standard Test Method for Tensile Properties of Plastics, ASTM International, West Conshohocken, PA.

10. Stratasys, CatalystEx. 2015, Stratasys.

11. Tymrak, B.M., M. Kreiger, and J.M. Pearce, Mechanical properties of components fabricated with open-source 3-D printers under realistic environmental conditions. Materials \& Design, 2014. 58: p. 242-246.

12. Bagsik, A., V. Schöppner, and E. Klemp, FDM Part Quality Manufactured with Ultem*9085 in Polymeric Materials 2010. 2010.

13. Dowling, N.E., Mechanical Behavior of Materials (4th Edition). 4th ed. 2012: Pearson. 


\section{List of figures}

Figure 1: Tensile testing specimen (a) CAD file, (b) 3D printed specimen (scale bar $=10 \mathrm{~mm}$ ).

Figure 2: Creep testing specimen (a) CAD file, (b) 3D printed specimens, where B, C, A marked on the specimens showing the $0^{\circ}, 45^{\circ}$, and $90^{\circ}$ printing orientations, respectively (scale bar $=10 \mathrm{~mm})$.

Figure 3: Fatigue testing specimen. (a) CAD file, (b) 3D printed specimen (scale bar $=20 \mathrm{~mm}$ ).

Figure 4: 3D printed ABS specimen is subject to tension in the creep tester. Horizontal cracks are visible on the specimen surface.

Figure 5: Stress-strain curves of three printing orientations.

Figure 6: Printing layer orientation variation with loading axis in tensile specimen.

Figure 7: Optical images of 0 o tensile bar (a) before the tensile test, (b) after the tensile test, (c) optical image of the cross-sectional view of the fracture surface (scale bar $=10 \mathrm{~mm}$ ).

Figure 8: Creep elongation vs. time of three printing orientations.

Figure 9: $S-N$ curve in log scale.

Figure 10: Optical images of the cross-sectional view of the fatigue test specimens: (a) $30 \mathrm{~N}$, (b) $40 \mathrm{~N}$, (c) $50 \mathrm{~N}$, (d) $60 \mathrm{~N}$ load (scale bar $=10 \mathrm{~mm}$ ) 


\section{List of tables}

Table 1: Averaged mechanical strength as a function of printing orientation

Table 2: Calculated $k$ values verses printing orientation 